\title{
FACTORS INFLUENCING READING PREFERENCE FOR OCCLUDED SINGLE COMPOSITION STRUCTURE CHARACTERS
}

\author{
Ching Chih Liao \\ Department of Commercial Design, Ming Chuan University \\ 5 De Ming Rd., Gui Shan District, Taoyuan City 333, Taiwan
}

\begin{abstract}
Past studies have discovered that that occlusion on the right (top-right, bottom-right) or on the bottom yields faster recognition time and higher reading accuracy for Chinese characters composed of two or three radicals, regardless of vertical or horizontal arrangement, or occluding one or two sides. In order to investigate whether occlusion of single composition structure characters, in the absence of accompanying parts, would yield similar recognition results, this pilot study was undertaken. Given the hypothesis that different positions of occlusion applied to numerals and Chinese characters would yield different reading preference results, this research selected nine each of Chinese characters and Arabic numerals, occluding each at the top, bottom, left, and right, comprising 72 samples. Coming from design and non-design education backgrounds, 160 participants were recruited to investigate how and why they would rank the modes of occlusion for preference. Using nonparametric tests to analyze the experimental results revealed a significant difference among the occlusion position for numbers and Chinese characters. Chinese characters occluded on the left were most preferred, those with right occlusion least. Numbers occluded on the bottom were most preferred, those with right occlusion least. The primary reason given for preference, for both Chinese characters and numbers, was ease of recognition and visual balance. The main reason for not preferring either occluded Chinese characters or numbers was that they were not easily recognized, seemed unbalanced and non-aesthetically pleasing. Regarding differing education background of the participants, there was significant difference for the Chinese characters occluded at the top and bottom, and non-significant difference for reading preference of occluded numerals.
\end{abstract}

\section{KEYWORDS}

Single Composition Structure Chinese Characters, Arabic Numerals, Reading Recognition (Recognition), Reading Preference, Balance

\section{INTRODUCTION}

The primary function of written words is to communicate and transmit messages, thus clear and complete words are most recognizable. However, in visual communication, words not only serve to transfer information, but are sometimes treated as processed reading images (Leeuwen, 2005) and even used to express emotions through different forms of arrangement (Malik, Aitken \& Waalen, 2016). All of these serve to achieve certain results such as increasing attention and memory. Focusing discussion on recognition and preference regarding occluded words aims to provide relevant research data which is beneficial to designers. When this is applied to graphic design, product design, or 3-D package design, designers can quickly decide on the positions of occlusion, proportions of occlusion and modes of occlusion, flattening their learning curve and decreasing uncertainty.

Luijkx, Thillou, \& Gosselin (2006) defined incomplete words as unknown and unseen words, meaning parts of letters have been cut or occluded. Simplifying Chinese character strokes is not a modern design expression, but something that can be traced back to Emperor Qianlong of Ching Dynasty who, on a trip to the region south of the Yangtze River, was strolling around Mid-lake Pavilion on West Lake at night. He was fascinated by the beautiful landscape and carved 虫 - - (worm, two) on the stele of the pavilion to commemorate the unbounded scene by eliminating the outline borders of two Chinese characters 風月 (scenery = wind, moon). In the European language world, application of occlusion to design is common. One designer 
occluded parts of letters in the activity theme on a poster to imply that by people's neglectfulness, valuable history may die with time. From the above description, we can learn that processed words can transmit artistic concepts, increase delight, and stir up emotional reactions. From a semiotics perspective, the unique letters on the stele are able to form a typographic landscape (Järlehed \& Jaworski, 2015). Due to different font features such as serifs, proportions, construction, organization, cohesion, etc. compared to texts, fonts can generate more of a local culture connection, a semiotic resource (Pescatore Frisk \& Pauwels, 2019). Placing incomplete words or punctuation on a poster can connect intended meaning of the theme of an activity with an even more powerful appeal.

In their study, Tseng, Chang \& Wang (1965) noted that words possess a certain amount of information and redundancy. Thus, this exploration on how far characters can be simplified and still retain reading recognition is undertaken. In related studies, the author of this paper (Liao, 2018; Tsao \& Liao, 2015) also discovered that when Chinese characters composed of two and three radicals are partially occluded at appropriate positions, it does not affect recognition. This corresponds to the viewpoints of recognition by components theory and object simplification (Biederman, 1981, 1987). Thus, we can conclude that application of occluded characters in practical design is a workable technique, but the prerequisite is that we must first understand the construction of character composition, preserve the recognizable and crucial starting strokes and radicals, and apply the appropriate proportions of occlusion. However, Chinese characters are very complicated. The key to recognition of occluded Chinese characters that are composed of two and three radicals is in their top-left starting strokes; therefore, right occlusion (including top-right and bottom-right occlusion) has the least effect on recognition. Yet, is this so for a character composed of a single radical? Thus, this pilot study undertakes to understand factors influencing participants' reading preference for occluded single radical characters as to follow-up on discussion of the effects of their occlusion on reading recognition results.

\subsection{Composition Structures of Traditional Chinese Characters and European Text Fonts}

Traditional Chinese characters are primarily composed of recognized components and radicals. From the perspective of Chinese character design, they can be divided into nine forms: 1 . One character which cannot be disassembled, such as 米 (rice); 2. Composed of right and left parts, such as 林 (forest); 3. Composed of right part with top-bottom radicals and left as a whole, such as 結 (tie); 4. Composed of left part with top-bottom radicals and right as a whole, such as 部 (part); 5. Composed of top and bottom parts, such as 歪 (crooked); 6. Composed of top part with left-right radicals and bottom as a whole, such as 然 (just so); 7. Composed of bottom part with left-right radicals and top as a whole, such as 蔽 (cover); 8. Composed of top, middle, bottom parts, such as 葉 (leaf); 9. Composed of left, middle, right parts, such as 邀 (invite) (Chen, 1974; Wu \& Chiu, 1995). Besides these compositions of recognized components and radicals, Chinese characters also involve strokes, 26 kinds of strokes in all, which are mainly composed of various horizontal and vertical strokes, wherein horizontal strokes account for the majority (Peng, 1982).

Words in European languages are primarily composed from among the 26 English letters. In typography, a font set in European languages mostly includes English letters and numerals, the design of which can be divided into six types (Zhu, 2009): Old Style, Sans Serif, Slab Serif, Script, Modern, and Decorative (also known as Baskerville). Sans serif fonts, because of having no extending feature at the end of strokes, are simple, clean, and modern, with Helvetica as a representative. Slab Serif fonts, wherein strokes contain slab angular serifs and transitions with back-in-time features, are good for headlines, as represented by Memphis. In general, Arabic numerals and English letters comprise vertical, horizontal, slash, and curve strokes. Aside from 4 and 5 , all other numbers can be completed in one stroke. The complexity of stroke and directionality for Chinese characters is higher than for Arabic numerals, even those with single structure composition such as 天 (sky).

\subsection{Aesthetic Features of Chinese Characters}

The classical concept of beauty is that it is a coherent unity of parts composing a pleasing aesthetic whole based on proportion, harmony, symmetry, and similar perceptions (Zalta, 2004; Ranscombe, Hicks, Mullineux \& Singh, 2012). Sun, Lian, Tang, \& Xiao (2015) tried to measure the aesthetic perception of Chinese handwriting using artificial intelligence and proposed three aspects for these measures. First, alignment and 
stability: Whether the composition of strokes is a vertical composition structure, horizontal composition structure, or surrounding composition structure, it must attain structural balance. Second, white space ratio (WSR): This illustrates that Chinese character strokes stress density; that is to say, the WSR cannot be too dispersed or too crowded. Third, gap between strokes: The means of composition between radicals involves stroke directions and positions (Xu, Jiang, Lau, \& Pan, 2007) and gap dimension also affects the determination of aesthetic perception. From the aforementioned, we can see a balanced and stable overall structure, tight composition between strokes and radicals, and degree of density will all affect the decision as to whether a Chinese character is aesthetically pleasing or not.

\subsection{Preference}

Within psychology, preference is used to indicate an individual's attitude toward a person or an object (Lichtenstein \& Slovic, 2006), or to express an evaluative judgement of like or dislike for a person or an object (Scherer, 2005). Preference is not stable; the decision-making process can significantly alter preference (Brehm, 1956; Sharot, De Martino \& Dolan, 2009). Moreover, emotional factors have an important influence on preference formation and maintenance (Zajonc \& Markus, 1982). From the literature, we understand that geographic location, cultural background, religion, education, and upbringing can all affect one's preferences. Besides continuing to investigate recognition of processed and incomplete characters (Liao, 2019), the motivation behind this research is to more deeply explore whether or not partially occluded character structure, that leads to aesthetic features such as seeming imbalanced and not stable, would affect research participant's preferences.

\subsection{Comparison of Recognition between Those with Different Education Backgrounds}

The literature indicates that individuals with design background can sometimes demonstrate different degrees of recognition in the same circumstance. For example: Eisner (1994) mentioned that individuals with art education background can accept more diversified objects as they are also encouraged to innovate and trained to think more with their right brains (mainly responsible for visual space) (Chiang \& Sun, 2014). Thus, individuals with design background prefer visual thinking. Hsu \& Wang $(2009,2010)$ discovered that individuals with non-design education training prefer figurative images more than those with design background. Liao (2018) discovered that individuals with non-design education background are faster on occluded Chinese character response time.

Herewith, a primary purpose of this study is to discuss the preference reaction to occluded characters by individuals with different education backgrounds. One reason is that understanding the recognition difference among those with different education backgrounds allows designers to avoid being caught in subjective thinking. The second reason is that the results of this preference pilot study will be useful for deepening future reading recognition research and these results can also be beneficial in future design applications. Therefore, two hypotheses are proposed.

Hypothesis 1: When characters are occluded in different positions, the basic factor affecting preference for these occluded characters is that they should be recognizable.

Hypothesis 2: Reading preference results for different positions of occlusion is not the same for numerals and Chinese characters.

\section{Method}

\subsection{Experimental Design}

This study investigated two independent variables - type of text (Chinese characters and Arabic numerals) and occlusion position (top, bottom, left, right) - according to the dependent variable of participants' reading preference, using a nonparametric Friedman test to analyze the experimental results. Two sets of participants from design and non-design education backgrounds, 160 in all, were recruited to investigate their ranking order, 
and the reason for the ranking choices, of reading preference for four-occlusion positions applied to the selected texts. Each participant observed nine each of Chinese characters and numerals, for a total of 18, which were respectively each occluded in four different positions, comprising 72 samples.

\subsection{Participants}

From among students at two universities in Taiwan, 160 participants were recruited, consisting half from each of design and non-design backgrounds. The so-called design background comprises students from the departments of Graphic design, Industrial design and Digital media design, because they took basic design courses in the first year, including Design theory, Color Science and Basic design. There were 88 males and 72 females, with mean age of 25.3 years, standard deviation of 17.63 years.

\subsection{Producing the Stimulus Materials}

From the list of 4,000+ most common Chinese characters resulting from Wu's (1987) study, nine single composition Chinese characters, of the 1000 to 9649 frequency levels, were selected as stimuli, including 出 (exit), 不 (no), 大 (big), 了 (understand), 天 (sky), 回 (return), 心 (heart), 之 (a possessive participle), 上 (top). According to the literature, Mingti (明體) is one of the easier-to-recognize Chinese fonts (Shieh, Chen \& Cuang, 1997; Cai, Chi \& You, 2001; Dobres, Chahine, Reimer \& Mehler, 2014); as practical design technique for occluded characters is usually applied to large and bold characters to make it stand out and increase attention, bold Mingti font was selected for producing the design samples.

In previous pilot study results, Tsao \& Liao (2015) found that when a character is occluded on top, bottom, right, and left positions by $2 / 9$ proportion on each, it is not only recognizable but has the most obvious occlusion effect. Accordingly, 2/9 occlusion was applied on all four sides of each character, generating four samples for each of all nine selected Chinese characters, comprising the 36 samples shown in the left-hand columns of Table 1.

As for occlusion design of Arabic numerals, because of the long, narrow appearance, with width considerably less than height, and the stroke of the numeral 1 being too simple that occlusion has no effect, it was skipped. Thus the numerals 0 and 2 9 were used, to which Arial Unicode MS 300pt English font (a sans serif font just as Helvetica is) was applied to design samples, displaying the features of simple, clean, and modern. Due to their long and narrow appearances, the exact same occlusion principles used for Chinese characters cannot be applied to Arabic numerals. Rather, numeral width was divided into five equal parts and height into six equal parts. Occlusions of $1 / 5$ on the width and 1/6 on the height were pre-tested. Based on the pre-test results, the actual experiment proceeded with slightly adjustments to occlusion proportions for the design samples as shown in the right-hand columns of Table 1.

Samples of one character or one numeral occluded at top, bottom, left, and right were placed on the same screen. Each occlusion sample appeared in black font on a white background and in a $12 \times 12 \mathrm{~cm}$ square on a black background. A sequence of 18 screens were displayed through a self-developed program. Each sample was designed with pull-down ranking choices and a space for participants to note their reason(s) for the most and least preferred.

Table 1. Samples of Single Composition Structure Characters and Different Occlusion Positions

\begin{tabular}{|c|c|c|c|c|c|c|c|}
\hline \multicolumn{4}{|c|}{ Chinese Characters } & \multicolumn{4}{|c|}{ Numerals } \\
\hline $\begin{array}{l}\text { Occluded } \\
\text { Top }\end{array}$ & $\begin{array}{l}\text { Occluded } \\
\text { Bottom }\end{array}$ & $\begin{array}{c}\text { Occluded } \\
\text { Left }\end{array}$ & $\begin{array}{l}\text { Occluded } \\
\text { Right }\end{array}$ & $\begin{array}{l}\text { Occluded } \\
\text { Top }\end{array}$ & $\begin{array}{l}\text { Occluded } \\
\text { Bottom }\end{array}$ & $\begin{array}{l}\text { Occluded } \\
\text { Left }\end{array}$ & $\begin{array}{l}\text { Occluded } \\
\text { Right }\end{array}$ \\
\hline 亖 & 4 & H & 出 & U & ก & 0 & $C$ \\
\hline$\uparrow$ & 不 & 不 & 不 & $\angle$ & 7 & 2 & 2 \\
\hline 大 & 大 & 大 & 大 & 3 & .3 & 3 & 3 \\
\hline 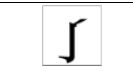 & 阝 & $\sqrt{3}$ & $\sqrt{5}$ & 4 & 4 & 4 & 4 \\
\hline 大 & 天 & 天 & 天 & 5 & .5 & 5 & 5 \\
\hline
\end{tabular}




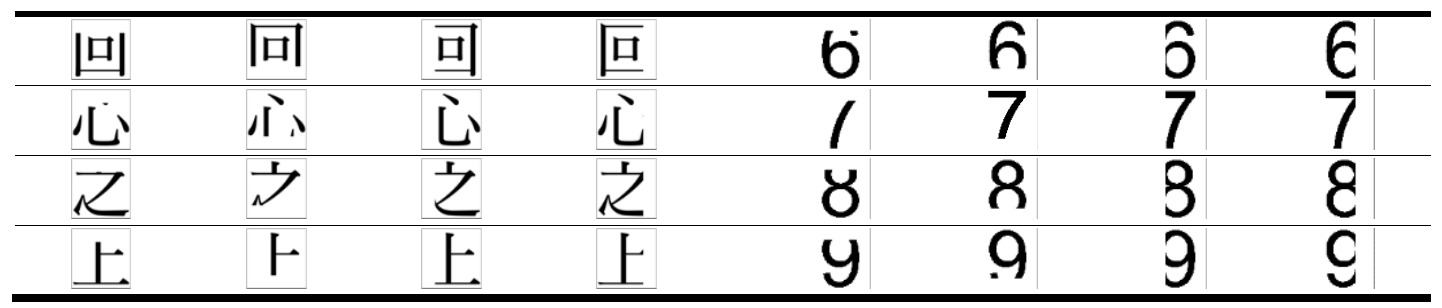

\subsection{Experimental Procedures}

Each of the 160 participants ranked each of the four occlusion positions on the same screen for all of the numerals and characters with 1 as the most preferred to 4 as the least preferred; moreover, participants wrote reason(s) for their order of preference. To avoid the learning effect on experimental results, the display order for numerals and characters was switched after each participant. The stimulus materials of the nine Chinese characters and nine numerals were randomly displayed. The test took approximately 20 minutes; each participant completed it independently and received a gift upon completion.

\subsection{Results}

The data was analyzed by nonparametric K-related-samples test. Results showed that $\chi^{2}(3)=126.07$ with $p<.001$ for Chinese character samples and indicated that all participants had at least one significantly different reading preference for one occlusion position among the four tested. Data was further analyzed by Two-Related-Samples Tests, revealing a rank of preference for occluded positions on Chinese characters as left, bottom, top, right, with left-side occlusion having highest preference and right-side occlusion as the least preferred.

Analysis of numeral samples showed that $\chi^{2}(3)=278.07$ with $p<.001$, which also indicated that all participants had at least one significantly different reading preference for one occlusion position among the four. Again, data was further analyzed by Two-Related-Samples Tests, showing a rank of preference for occluded positions on numerals as bottom, top, left, right, meaning bottom occlusion has highest preference and right-side occlusion was least preferred.

The results between two different education background groups for Chinese characters occluded on the top yielded $\chi^{2}(1)=5.872$ and $p=.01$, and for bottom-position occlusion $\chi^{2}(1)=13.69$ and $p=.001$. This indicates that individuals with different education backgrounds had significantly different reading preferences for certain characters being occluded in different positions. Participants with non-design background had a higher reading preference than those with design background for Chinese characters such as 出 (exit), 大 (big), 回 (return) being occluded on top; and individuals with design background had a higher reading preference than those with nondesign background for Chinese characters 天 (sky), 上 (top) being occluded on bottom. There was no preference difference between the different education backgrounds for numerals occluded in different positions.

\subsubsection{Preference Differences for Chinese Characters Being Occluded in Four Positions and Reasons}

In order to further understand whether or not participants had significantly different preferences among the occlusion in four positions for the nine Chinese characters, analysis by nonparametric K-related-samples test was used. For Chinese characters, occlusion at top, bottom, left and right positions yielded the following results: $\chi^{2}(8)=742.76, p<.001 ; \chi^{2}(8)=523.22, p<.001 ; \chi^{2}(8)=490.18, p<.001 ; \chi^{2}(8)=460.29, p<.001$, respectively (Table $2)$. This indicates that at least one Chinese character among the nine differed significantly when occluded in each position.

As shown by the mean rank in Table 2, when Chinese characters were occluded on top, if the starting stroke was from the top and was a horizontal stroke such as 不, 了, 天, least preference was observed due to the occluded character not being recognized easily, according to reasons provided by the participants. On the other hand, if the starting stroke was not from the top, the reading preference was not easily affected and this position of occlusion had a higher preference, such as for 出, 上. When Chinese characters were occluded on bottom, if 
a primary stroke was a horizontal stroke and was on the lowest part of the character, such as for 上, this was the hardest to recognize and had low preference.

When Chinese characters were occluded on left, and the core of the stroke was toward the right, such as $了$, this did not affect recognition and had a higher preference. But if the core of the strokes and features were preserved when occluded on the right, such as for $之$, this did not affect recognition and had a higher preference. It is also worth mentioning that preference was low for right- or left-side occlusion of characters with bilateral symmetry such as 出 and 回, because of visual imbalance.

A list of reasons for preference and non-preference was collated from the participants' feedback. Examples of preference reasons that appeared most frequently included: easiest recognition, character appears very stable, recognize it at a glance, character can be recognized, no problem in recognition, and close to the complete character. Non-preference reasons were: hard to recognize, aesthetic sense is spoiled, mistaken for another character, uncomfortable to look at, and low level of stability.

Table 2. Comparison among Chinese Characters Being Occluded in Four Positions

\begin{tabular}{lrrrrrrrrr}
\hline & 出 & 不 & 大 & 了 & 天 & 回 & 心 & 之 & 上 \\
\hline Occluded Top & $\mathbf{2 . 5 5}$ & $\mathbf{7 . 0 8}$ & $\mathbf{3 . 1 3}$ & $\mathbf{7 . 0 4}$ & $\mathbf{7 . 2 2}$ & 4.41 & 4.68 & 6.02 & $\mathbf{2 . 8 8}$ \\
\hline Occluded Bottom & 4.83 & $\mathbf{2 . 5 8}$ & 6.04 & 5.07 & 5.28 & $\mathbf{3 . 0 2}$ & $\mathbf{3 . 7 8}$ & $\mathbf{6 . 9 4}$ & $\mathbf{7 . 4 7}$ \\
\hline Occluded Left & 6.83 & 5.19 & $\mathbf{6 . 0 6}$ & $\mathbf{2 . 2 9}$ & 3.48 & $\mathbf{6 . 6 0}$ & 6.04 & 3.46 & 5.05 \\
\hline Occluded Right & $\mathbf{7 . 0 9}$ & 4.9 & 5.36 & 4.62 & $\mathbf{2 . 8 9}$ & 6.44 & $\mathbf{6 . 2 3}$ & $\mathbf{2 . 5 5}$ & 4.91 \\
\hline
\end{tabular}

Note: The numbers in each column represent the mean ranks. The lower the mean rank, the higher the preference.

\subsubsection{Preference Differences for Numerals Being Occluded in Four Positions and Reasons}

Nonparametric K-related-samples test was used to analyze results; numerals occluded on top, bottom, left and right positions yielded the following results: $\chi^{2}(8)=742.76, p<.001 ; \chi^{2}(8)=520.42, p<.001 ; \chi^{2}(8)=334.63$, $p<.001 ; \chi^{2}(8)=305.38, p<.001$, respectively (Table 3 ). This indicates that at least one numeral among the nine differed significantly when occluded on the top position.

As shown by the mean rank in Table 3, when numerals were occluded on top, and the core stroke was a horizontal one from the top such as 7, this was least preferred and most difficult to recognize. On the other hand, if the starting stroke was not from the top, the reading preference for occlusion at the top was less affected and received higher preference, such as for 9 and 0 . When numerals were occluded on bottom, and the core stroke was a horizontal one on the lowest part, such as for 2 , the preference was low due to not being easily recognized. Otherwise, if a vertical stroke was involved and the numeral was occluded on the bottom, the reading preference was not affected much, such as for 6 and 7, thus yielding a higher preference.

When numerals were occluded on the left, and the core stroke and features were on the left, such as for 6 and 4, recognition was affected and preference was lower. But if the core stroke was on the right, such as for 7 , the reading preference for occlusion on the left was not affected and preference was higher. When numerals such as 2 and 4 were occluded on the right, because the right-side feature was not occluded, higher preference was observed. Similar to Chinese characters, due to visual imbalance when bilaterally symmetric numerals such as 0 and 8 were occluded on the right or left, this was not preferred at all.

Reasons for preference that appeared the most in feedback from participants included: stylish, clear, numeral appears very stable, easy recognition, comfortable to look at, and close to a complete numeral. Non-preference reasons included: not aesthetically pleasing, aesthetics are spoiled, easy to be mistaken for another numeral, uncomfortable to look at, and balance is lost.

Comparing the preference descriptions between Chinese characters and numerals, the most frequently mentioned description for characters was reading recognition is easy, while for numerals the most frequent phrases were in regard to aesthetic sense and having stylish design features. 
Table 3. Comparison among Numerals Being Occluded in Four Positions

\begin{tabular}{llllllllll}
\hline & $\mathbf{0}$ & $\mathbf{2}$ & $\mathbf{3}$ & $\mathbf{4}$ & $\mathbf{5}$ & $\mathbf{6}$ & $\mathbf{7}$ & $\mathbf{8}$ & $\mathbf{9}$ \\
\hline Occluded Top & $\mathbf{3 . 7 4}$ & 4.35 & $\mathbf{4 . 6 1}$ & $\mathbf{6 . 8 2}$ & $\mathbf{5 . 8 9}$ & 4.74 & $\mathbf{7 . 7 4}$ & 4.52 & $\mathbf{2 . 5 9}$ \\
\hline Occluded Bottom & 4.52 & $\mathbf{8 . 0 5}$ & 5.22 & 3.90 & 4.80 & $\mathbf{3 . 7 1}$ & 3.87 & $\mathbf{4 . 3 1}$ & $\mathbf{6 . 6 3}$ \\
\hline Occluded Left & 5.48 & 3.44 & 5.08 & 6.00 & 5.38 & $\mathbf{6 . 3 8}$ & $\mathbf{2 . 4 1}$ & 5.52 & 5.32 \\
\hline Occluded Right & $\mathbf{6 . 5 8}$ & $\mathbf{3 . 2 3}$ & $\mathbf{5 . 7 1}$ & $\mathbf{3 . 2 1}$ & $\mathbf{4 . 2 3}$ & 5.22 & 5.11 & $\mathbf{5 . 9 7}$ & 5.74 \\
\hline
\end{tabular}

Note: The numbers in each column represent the mean ranks. The lower the mean rank, the higher the preference.

\section{DISCUSSION AND CONCLUSION}

Through preference rank analysis of single composition structure Chinese characters and Arabic numerals occluded in different positions, it is discovered that characters and numerals with bilateral symmetrical structures are both less preferred when the right or left part is occluded. According to the reasons given by study participants, aside from the main reason of not being easily recognized, the low preference arises from feelings about aesthetic sense. When this type of text is partially occluded either on the right or the left, balance is lost, thus it lacks the sense of being aesthetically pleasing. The Chinese character 天 is an exception; although it is bilaterally symmetric, when partially occluded either on the right or the left, it still preserves its structural wholeness and stroke density, thus recognition and balance are maintained and preference is unaffected. The numeral 9 only has a higher reading preference when occluded on top and low preference when other positions are occluded. It is deduced that top, left and right occlusion of this numeral affects its recognition and balance.

Thereunder, the primary reason for occluded character preference is shown to be recognition, followed by aesthetic sense of balance. The study results demonstrate that occlusion in different positions indeed affects preference and having design background or not only slightly affects reading preference for Chinese characters, with no preference difference for Arabic numerals. Moreover, the results of this study demonstrate that the effect of position of occlusion on reading preference and ease of recognition for single composition structure characters both differ from that of characters composed of two and three radicals. Future research will aim to further pursue the study of recognition for occluded single composition structure characters, to verify these results. Meanwhile, the outcomes of this study are sufficient to serve as an auxiliary reference and an application basis for design practices.

\section{ACKNOWLEDGEMENT}

This study was supported by Ministry of Science and Technology in Taiwan, under the grand numbers of MOST 108-2410-H-130 -010.

\section{REFERENCES}

Biederman, I., 1981. On the semantics of a glance at a scene. In Kubovy, M. \& Pomerantz, J.R. (Eds.), Perceptual organization (pp.213-253). Hillsdale, NJ: Erlbaum.

Biederman, I., 1987. Recognition-by-Components: A Theory of Human Image Understanding. Psychological Review, 94(2), 115-147.

Brehm, J.W., 1956. Post-decision changes in desirability of choice alternatives. Journal of Abnormal and Social Psychology, 52, 384-389.

Chen, X.R., 1974. Graphic design of English and Chinese characters. Taipei, Taiwan: Dong-Woo Printing. (In Chinese)

Ching, S.B. \& Sun, C.W., 2014. Impacts of learning background and different contextual sentences on shot interpretation in word-to-image transcoding process. Journal of Design, 19 (3), 41 - 62.

Eisner, E.W., 1994. Cognition and curriculum reconsidered (2nd ed), New York: Teachers College Press.

Hsu, C.C. \& Wang, R.W.Y., 2009. The relationship between learning background and the preference to simplified graphics. Research in Arts Education, 17, 109 - 130. 
Hsu, C.C. \& Wang, R.W.Y., 2010. The relationship between shape features and degrees of graphic simplification. Journal of Design, 15(3), 87 - 105.

Järlehed, J. \& Jaworski, A., 2015. Typographic landscaping: Creativity, ideology, movement. Social Semiotics, 25(2), $117-125$.

Leeuwen, T.V. (2005). Typographic meaning, visual communication 4(2), 137-143.

Liao, C.C., 2018. Double-Sided Occluded Chinese Character Recognition Accuracy and Response Time for Design and Nondesign Educational Background. SAGE Open, 8(4), 2158244018810065.

Liao, C.C., 2019. Preference Impact of Diverse Modes of Occlusion on Single Composition Structure Chinese Characters and Arabic Numerals, In Proceeding of International Conference on Advanced Research in Applied Science and Engineering, Amsterdam, Netherlands. ISBN: 978-609-8239-71-3

Lichtenstein, S. \& Slovic, P., 2006. The construction of preference. New York: Cambridge University Press.

Luijkx, A. et al, 2006. Aprolongation-based approach for recognizing cut characters. Computer Vision and Graphics. Computational Imaging and Vision, 32, 1125-1130.

Malik, S. et al, 2016. Communicating Emotion With Animated Text, Visual Communication, 8(4), 469-479.

Peng, R.X., 1982. A preliminary report on statistical analysis of the structure of Chinese characters. Acta Psychologic Sinica, 385-390.

Pescatore Frisk, R. \& Pauwels, L., 2019. The shared stories that written words tell when no one is reading: exploring modality in typographic landscapes as an ecosocial semiotic system. visual communication, 1-34. doi:10.1177/1470357219860086

Ranscombe, C. et al, 2012. Visually decomposing vehicle images: Exploring the influence of different aesthetic features on consumer perception of brand. Design Studies, 33(4), 319-341. doi:10.1016/j.destud.2011.06.006

Scherer, K.R., 2005. What are emotions? And how can they be measured? Social Science Information, 44, 695-729.

Sharot, T. et al, 2009. How choice reveals and shapes expected hedonic outcome. Journal of Neuroscience, 29, 3760-3765.

Sun, R. et al, 2015. Aesthetic Visual Quality Evaluation of Chinese Handwritings. Proceedings of the Twenty-Fourth International Joint Conference on Artificial Intelligence (IJCAI 2015), Buenos Aires, Argentina, pp. 2510-2516.

Tsao, Y.C. \& Liao, C.C., 2015. Effect of structural composition and position of occlusion on the legibility of occluded Chinese characters. Perceptual \& Motor Skills, 120(2), 560-575.

Tseng, S.C. et al, 1965. An informational analysis of the Chinese language: The reconstruction of the removed strokes of the ideograms in printed sentence-texts. Acta Psychologic Sinica, 281-290.

Wu, J.T., 1987. [Exploring the phonetic and semantic features of Chinese words: Part 1. Frequency count of Chinese words, characters, and monosyllables]. (Grant No. NSC-75-0301-H002-24) Taipei, Taiwan: National Science Council. [in Chinese]

Wu, M.S. \& Chiu, I.T., 1995. Graphic design of Chinese character formation. Taipei, Taiwan: New Image. (In Chinese)

$\mathrm{Xu}, \mathrm{S}$. et al, 2007. An intelligent system for Chinese calligraphy. In Proceedings of the 22nd National Conference on Artificial Intelligence, Vancouver, British Columbia, Canada, vol. 2, pp.1578-1583.

Zajonc, R.B. \& Markus, H., 1982. Affective and cognitive factors in preferences. Journal of Consumer Research, 9(2), 123-131.

Zalta, E.N. (ed.) 2004. Stanford Encyclopedia of Philosophy. Stanford, CA: The Metaphysics Research Lab.

Zhu, Q. M., 2009. Graphic design (Typography). Publishing house: Longxi Books. 